\title{
Late oral acetaminophen versus immediate surgical ligation in preterm infants with persistent large patent ductus arteriosus
}

\author{
Sally Mashally, MD, ${ }^{\mathrm{a}}$ Lynne E. Nield, MD, ${ }^{\mathrm{a}, \mathrm{b}, \mathrm{c}}$ Patrick J. McNamara, MD, MSc, , , ,d,e \\ Fernando F. Martins, MD, PhD, ${ }^{\mathrm{f}}$ Afif El-Khuffash, MD, ${ }^{\mathrm{g}, \mathrm{h}}$ Amish Jain, MD, ${ }^{\mathrm{b}, \mathrm{c}, \mathrm{i}}$ and \\ Dany E. Weisz, MD, MSc ${ }^{\mathrm{a}, \mathrm{b}, \mathrm{c}}$
}

\section{ABSTRACT}

Objective: The study objective was to evaluate the association of oral acetaminophen therapy versus immediate surgical ligation with neonatal outcomes in infants with persistent patent ductus arteriosus.

Methods: We performed a retrospective cohort study of preterm infants born $28^{+6}$ weeks or less gestational age with persistent large patent ductus arteriosus being considered for surgical ligation after unsuccessful medical therapy. Infants in epoch 1 (July 2009 to June 2012) were immediately referred for ligation, and infants in epoch 2 (July 2012 to June 2015) were treated with oral acetaminophen and referred for ligation in the absence of improvement. The primary outcome was a composite of death or chronic lung disease.

Results: A total of 92 infants with median (interquartile range) gestational age 25.2 weeks (24.4-26.3) had persistent large patent ductus arteriosus (43 in epoch 1,49 in epoch 2). Infants in epoch 2 had decreased surgical ligation (26 [53\%] vs 31 [72\%]; adjusted odds ratio [aOR], 0.32; 95\% confidence interval [CI], $0.12-0.89$ ) but increased chronic lung disease (36 [73\%] vs 25 [58\%]; aOR, 3.34; 95\% CI, 1.05-10.58) and increased death/chronic lung disease of borderline significance (39 [80\%] vs 29 [67\%]; aOR, 3.09; 95\% CI, 0.99-9.63). Infants in epoch 2 took longer to wean off of positive pressure ventilation (28.5 vs 24 days after enrollment; aOR, 0.52; 95\% CI, 0.31-0.85).

Conclusions: Late oral acetaminophen therapy for infants with persistent patent ductus arteriosus is associated with reduced surgical ligation but increased chronic lung disease. In light of a lack of improvement in clinical outcomes, the individual contributory effects of acetaminophen, surgical ligation, and prolonged exposure to patent ductus arteriosus require further study to define the optimal approach. (J Thorac Cardiovasc Surg 2018;156:1937-44)

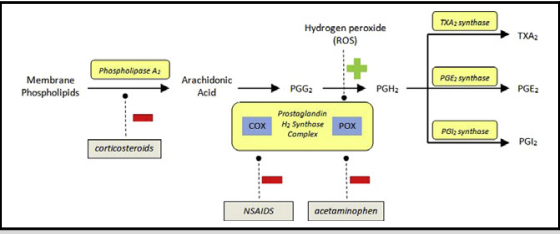

Effect of acetaminophen on enzymes that regulate prostaglandins promoting ductal patency. (Adapted with permission from Weisz D, Jain A, McNamara P. Patent Ductus Arteriosus. Buenos Aires, Argentina: Ediciones Journal SA; 2016.

\section{Central Message}

Late acetaminophen treatment for persistent patent ductus arteriosus in preterm infants reduces the need for surgical ligation but increases CLD and duration of respiratory support.

\section{Perspective}

Acetaminophen treatment is often administered to facilitate closure of persistent patent ductus arteriosus and avoid surgical ligation among extremely preterm infants. Our study suggests that acetaminophen treatment and avoidance of surgical ligation may be associated with adverse outcomes. Further study is required to determine which infants may benefit from earlier surgical ligation.

See Editorial Commentary page 1945.

See Editorial page 1935.
The management of patent ductus arteriosus (PDA), which occurs in up to $70 \%$ of infants born extremely preterm, is an area of significant controversy. ${ }^{1}$ Observational

From the ${ }^{a}$ Department of Newborn and Developmental Pediatrics, Sunnybrook Health Sciences Centre, Toronto, Ontario, Canada; Departments of ${ }^{b}$ Pediatrics and ${ }^{\mathrm{d}}$ Physiology, University of Toronto, Toronto, Ontario, Canada; ${ }^{\mathrm{c}}$ Department of Pediatrics, Hospital for Sick Children, Toronto, Ontario, Canada; ${ }^{\mathrm{e}}$ Physiology and Experimental Medicine Program, SickKids Research Institute, Toronto, Ontario, Canada; ${ }^{\mathrm{f}}$ Fernandes Figueira Institute, Oswaldo Cruz Foundation, Rio de Janeiro, Brazil; ' ${ }^{\mathrm{g}}$ Department of Neonatology, The Rotunda Hospital, Dublin, Ireland; ${ }^{\mathrm{h}}$ Department of Paediatrics, School of Medicine, Royal College of Surgeons in Ireland, Dublin, Ireland; and ${ }^{\mathrm{i}}$ Department of Pediatrics, Mt Sinai Hospital, Toronto, Ontario, Canada. studies have associated PDA ligation in very low birthweight infants with increased chronic lung disease (CLD), severe retinopathy of prematurity (ROP), and

Received for publication Nov 24, 2017; revisions received April 15, 2018; accepted for publication May 17, 2018; available ahead of print July 11, 2018.

Address for reprints: Dany E. Weisz, MD, MSc, Department of Newborn and Developmental Paediatrics Sunnybrook Health Sciences Centre, 2075 Bayview Ave, Toronto, Ontario, Canada, M4N 3M5 (E-mail: dany.weisz@sunnybrook.ca). $0022-5223 / \$ 36.00$

Copyright (C) 2018 by The American Association for Thoracic Surgery https://doi.org/10.1016/j.jtcvs.2018.05.098 

Abbreviations and Acronyms
$\mathrm{aOR}=$ adjusted odds ratio
$\mathrm{CI}=$ confidence interval
CLD $=$ chronic lung disease
COXI $=$ cyclooxygenase inhibitor
Fio2 = fraction of inspired oxygen
$\mathrm{GA}=$ gestational age
$\mathrm{IQR}=$ interquartile range
IV = intravenously
IVH = intraventricular hemorrhage
MAP $=$ mean airway pressure
NICU $=$ neonatal intensive care unit
$\mathrm{ROP}=$ retinopathy of prematurity

Scanning this QR code will take you to the article title page.

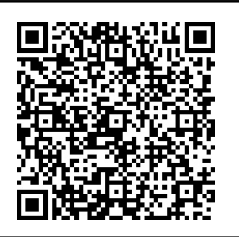

neurodevelopmental impairment in early childhood..$^{2-4}$ The publication of these reports has been associated with a secular change in clinical management toward avoiding or delaying PDA ligation., ${ }^{5,6}$ Although the precise causal mechanism of ligation and adverse outcomes remains uncertain, surgical complications such as left vocal cord paresis, postoperative hemodynamic compromise, or the effects of general anesthesia may contribute to increased morbidity.

Acetaminophen may be an effective alternative therapy to surgical ligation or conservative management for facilitating closure of persistent large PDA in preterm neonates being considered for surgical ligation after failure or contraindication of treatment with cyclooxygenase inhibitors (COXIs). The efficacy of acetaminophen for early closure of PDA has been demonstrated in several randomized controlled trials. ${ }^{7-10}$ Although uncontrolled studies have associated late acetaminophen therapy with ductal closure and avoidance of PDA ligation, ${ }^{11-14}$ the broader impact on neonatal outcomes of administering acetaminophen in lieu of immediate surgical ligation remains unknown.

The objective of this study was to evaluate the association of late oral acetaminophen therapy versus immediate surgical ligation for persistent large PDA with neonatal outcomes. Given the association between surgical ligation and adverse outcomes, and the efficacy of acetaminophen to facilitate ductal closure and avoid PDA surgery, we hypothesized that oral acetaminophen treatment would be associated with a reduction in morbidity.

\section{MATERIALS AND METHODS}

This was a retrospective comparative epoch cohort study of preterm infants born at gestational age (GA) $28^{+6}$ weeks or less with persistent large PDA being considered for surgical ligation after failure of COXI or conservative therapy.

The neonatal intensive care unit (NICU) at Sunnybrook Health Sciences Centre is a tertiary perinatal center that admits approximately 200 preterm infants (born $\leq 28$ weeks GA) annually. The standard of care in our unit is to treat extremely low birthweight infants $(<1000 \mathrm{~g})$ with prophylactic indomethacin $(0.1 \mathrm{mg} / \mathrm{kg}$ intravenously [IV] daily for 3 days), beginning within the first 6 hours of life. However in 2010-2011, a prolonged shortage of indomethacin resulted in its administration being temporarily restricted to infants born at GA 25 weeks or less. Beyond the transitional period, infants with clinically symptomatic PDA (eg, increasing ventilatory support, cardiac murmur, or wide pulse pressure) are evaluated by a neonatologist with expertise in targeted neonatal echocardiography and undergo an anatomic evaluation by a pediatric cardiologist. Up to 2 courses of COXI therapy (indomethacin [0.2 mg/kg IV every 12 hours for 3 doses] or ibuprofen $[10 \mathrm{mg} / \mathrm{kg} \mathrm{IV} /$ by mouth once, followed by $5 \mathrm{mg} / \mathrm{kg} \mathrm{IV} / \mathrm{by}$ mouth daily for 2 doses]) are administered to facilitate ductal closure in infants with clinically and echocardiographically significant PDA. The echocardiography criteria for large PDA include ductal diameter $2.0 \mathrm{~mm}$ or greater, an unrestrictive systemic-to-pulmonary shunt, signs of left heart volume loading and pulmonary overcirculation (eg, left ventricular dilatation, left atrium to aortic root ratio $\geq 1.5$ ), and diastolic flow reversal in the abdominal aorta. Echocardiography is performed using a Vivid E9 ultrasound machine (GE Healthcare, Chicago, Ill) and a $12 \mathrm{MHz}$ phased-array multi-frequency probe.

Infants with large PDA also may be simultaneously managed using targeted changes in mechanical ventilation to mitigate the ductal shunt, such as increased positive end-expiratory pressure to reduce alveolar edema or mild hypercarbic acidosis $(\mathrm{pH} 7.25-7.30$, partial pressure of carbon dioxide $50-60 \mathrm{~mm} \mathrm{Hg}$ ) to facilitate increased pulmonary arterial pressure). If COXI therapy is unsuccessful in facilitating PDA closure, then infants with radiographic pulmonary edema or echocardiography indices of left heart volume loading may undergo a trial of diuretic therapy.

Infants with persistent large PDA after unsuccessful pharmacologic or conservative therapy are considered for surgical ligation. The clinical criteria for surgical ligation include clinical signs of a large ductal shunt (eg, murmur, active precordium, radiographic pulmonary edema, or cardiomegaly) with concomitant respiratory insufficiency or end-organ hypoperfusion (eg, renal insufficiency, inability to tolerate increases in enteral feeding volume). Only infants who are dependent on invasive mechanical ventilation or noninvasive mechanical ventilation with high mean airway pressure (MAP) $\left(\geq 8 \mathrm{~cm} \mathrm{H}_{2} \mathrm{O}\right)$ or high fraction of inspired oxygen $\left(\mathrm{FIO}_{2}\right)$ and inability to wean are considered candidates for PDA ligation. Infants are formally referred for surgery, triaged according to clinical and echocardiography severity, ${ }^{15}$ and subsequently transported to our local quaternary center for the procedure. The operative approach includes a left lateral thoracotomy, and an intra- or extrapleural approach using a clip or ligature at the discretion of the attending surgeon.

In July 2012, oral acetaminophen was introduced at Sunnybrook Health Sciences Centre as a late therapy after failure or contraindication of COXI treatment in preterm infants with persistent large PDA being considered for surgical ligation. The goal of acetaminophen treatment was to promote sufficient ductal restriction and a reduction in left heart volume loading, pulmonary overcirculation, and systemic arterial diastolic flow reversal to effect clinical improvement and avoid ligation.

To evaluate the association of late oral acetaminophen therapy versus immediate surgical ligation with neonatal outcomes, we compared a recent 3-year cohort of infants (acetaminophen epoch; July 1, 2012, to June 30, 2015) with a prior historical cohort comprising infants with persistent large PDA (immediate surgical ligation epoch; July 1, 2009, to June 30, 2012). 
Both the acetaminophen and immediate surgical ligation groups comprised preterm infants $28^{+6}$ weeks or less GA in their respective epochs who were considered candidates for PDA ligation. Infants in the immediate surgical ligation epoch were referred for PDA surgery directly after failure of medical therapy. During the acetaminophen epoch, infants with persistent large PDA were treated with a 3- to 7-day course of acetaminophen administered orally at a dose of $15 \mathrm{mg} / \mathrm{kg}$ every 6 hours. ${ }^{14}$ The duration of the therapeutic course was at the discretion of the attending neonatologist. Infants with hepatic dysfunction, experiencing clinical deterioration (eg, sepsis) during the treatment or in whom surgical ligation was prioritized because of clinical instability may have received an acetaminophen course of less than 7 days duration. Infants underwent echocardiography within 3 days of completing acetaminophen treatment. Infants with persistent clinical and echocardiography signs of large PDA after completion of the treatment course were referred for surgical ligation.

Eligible infants were identified using local clinical and echocardiography databases, as all patients being considered for PDA ligation undergo serial echocardiography. Infants were excluded if they had chromosomal abnormalities or congenital heart disease (other than small secundum atrial septal defect or muscular ventricular septal defect).

Data on important antenatal and perinatal characteristics were abstracted from patient charts. To guide the appropriate collection of postnatal confounders, a date of study "enrollment" was determined for each infant. Infants in both epochs were considered "enrolled" on the earliest date that they were considered a candidate for surgical ligation. The enrollment date for infants in the immediate surgical ligation epoch was the date of referral for PDA surgery (formally documented in the patient chart and clinical database) and infants in the acetaminophen epoch were considered enrolled on the date of initiation of the course of acetaminophen.

Detailed collection of postnatal, pre-enrollment characteristics was performed to enable adjustment for indices of illness severity and morbidities of prematurity that arose or were acquired before enrollment. The preenrollment characteristics abstracted included the administration of prophylactic indomethacin, date and total dose of COXI, date of first clinical diagnosis of PDA, necrotizing enterocolitis stage 2 or greater (according to the modified Bell's staging), ${ }^{16}$ culture-positive sepsis (defined as early or late-onset clinical sepsis accompanied by a positive culture from a sterile site), severe pulmonary hemorrhage (defined as blood suctioned from the trachea or endotracheal tube accompanied by new or worsening respiratory failure necessitating the initiation or augmentation of invasive mechanical ventilation and increased $\mathrm{FiO}_{2}$ ), administration of inhaled nitric oxide, and inotropic support for systemic hypotension. The highest severity of intraventricular hemorrhage (IVH) or periventricular hemorrhage infarction and the presence or absence of cystic periventricular leukomalacia on the date of enrollment were noted.

We identified that dependence on mechanical ventilation was a potentially important confounder because it is strongly associated with surgical ligation and neonatal outcomes such as death, CLD, and ROP. As a result, we collected daily ventilation information (average daily MAP and most intensive mode of ventilation used) from birth to discharge or transfer to a level II NICU. Transfer to a level II NICU typically occurs after successful weaning from positive-pressure ventilation. For infants transferred to a level II NICU, the ascertainment of in-hospital survival and CLD was performed using discharge information provided by the level II unit.

Among infants treated with ligation, the frequency of surgical complications, including operative mortality, and postligation cardiac syndrome, was abstracted.

The primary outcome was a composite of death before discharge or moderate-severe CLD, defined as the need for supplemental oxygen or positive pressure ventilation at 36 weeks corrected GA. Secondary outcomes included surgical ligation, death before discharge, moderate-severe CLD, severe ROP requiring treatment with laser surgery or intravitreal vascular endothelial growth factor inhibitor, treatment with systemic corticosteroids, time to successful endotracheal extubation (defined as 7 days without reintubation), ${ }^{17}$ and time to wean off any positive pressure ventilation (invasive or noninvasive, defined as at least 7 days without reinitiation of positive pressure ventilation). An oxygen reduction test was not routinely performed in our unit.

We identified that a secular trend away from pharmacologic and surgical treatment of PDA may have resulted in selection bias in assembling the cohort of infants considered for ligation. Some infants among the broader cohort treated during the second epoch (2012-2015) may not have been considered for ligation (due to, for example, lower illness severity), but would have been considered for ligation in epoch 1 (2009-2012). To evaluate for potential selection bias, we compared the antenatal and postnatal clinical characteristics (including PDA diagnosis and treatment) among all infants in each epoch born at GA 28 weeks or less and admitted to the NICU.

\section{Statistical Analysis}

Descriptive statistics were used to summarize antenatal, perinatal, and pre-enrollment characteristics for infants in the 2 epochs. Mean (standard deviation) and median (interquartile range [IQR]) were used to characterize normally distributed and skewed data, respectively. The characteristics of the infants in each epoch were compared using the Student $t$ test or Wilcoxon rank-sum test for continuous variables and the chi-square test or Fisher exact test for categorical variables, as appropriate. Multivariable logistic regression analysis was used to estimate the association between epoch and neonatal outcomes, adjusting for perinatal and postnatal, preenrollment confounders. Cox proportional hazards modeling was used to estimate the association between epoch and time to successful extubation and time to successfully wean off all positive pressure ventilation. The final model was determined using bivariate screening, with variables retained if they resulted in a $7.5 \%$ change in the point estimate of the primary outcome. Goodness of fit was assessed using the Hosmer-Lemeshow statistic, and all models were evaluated for collinearity and overspecification. The proportional hazards assumption was verified using cumulative residual plots and examining log-negative log survival and cumulative hazard graphs.

Preliminary estimates indicated approximately 100 preterm infants were considered candidates for surgical ligation over the entire study period. With the presumption of a $70 \%$ event rate (death or CLD) in the immediate surgical ligation epoch, ${ }^{2}$ a 2 -sample, 2-sided test of proportions with $80 \%$ power and $5 \%$ type I error was estimated to detect a $20 \%$ absolute difference in the primary outcome.

Statistical analyses were performed using SAS version 9.4 (SAS Institute Inc, Cary, NC). All statistical tests were 2-sided with significance evaluated at the $5 \%$ level. This study was approved by the local hospital Research Ethics Board.

\section{RESULTS}

From July 1, 2009, to June 30, 2015, 92 infants born at $28^{+6}$ weeks or less gestation with persistent large PDA were considered candidates for surgical ligation after failure or contraindication to COXI or conservative therapy (43 in the immediate surgical ligation group and 49 in the acetaminophen group). Ductal diameter was $1.5 \mathrm{~mm}$ or greater, and the ductal shunt was unrestrictive in all infants, with $90 \%$ of infants having PDA diameter $2.0 \mathrm{~mm}$ or greater at the time of enrollment. There were no significant differences between the acetaminophen and immediate surgical ligation groups in their antenatal and perinatal characteristics, with the exception of known secular trends in clinical management (antenatal magnesium sulphate for 
TABLE 1. Perinatal and postnatal, pre-enrollment clinical, and echocardiography characteristics of preterm infants being considered for surgical ligation

\begin{tabular}{|c|c|c|c|}
\hline & Immediate surgical ligation epoch $(n=43)$ & Acetaminophen epoch $(n=49)$ & $P$ value \\
\hline \multicolumn{4}{|l|}{ Perinatal characteristics } \\
\hline GA at birth (wk) & $25.1(24.7-26.1)$ & $25.1(24.4-26.3)$ & .48 \\
\hline Birthweight (g) & $740(650-851)$ & $730(640-865)$ & .96 \\
\hline Small for GA (<10th percentile), n $(\%)$ & $4(9)$ & $5(10)$ & .88 \\
\hline Female sex, n $(\%)$ & $22(51)$ & $25(51)$ & .99 \\
\hline Outborn, n (\%) & $11(26)$ & $7(14)$ & .17 \\
\hline Multiple gestation, $\mathrm{n}(\%)$ & $10(23)$ & $20(41)$ & .07 \\
\hline Caesarean delivery, $\mathrm{n}(\%)$ & $29(67)$ & $32(65)$ & .83 \\
\hline Antenatal corticosteroids, $\mathrm{n}(\%)$ & $38(90)$ & $44(89)$ & .91 \\
\hline Magnesium sulfate for fetal neuroprotection, $\mathrm{n}(\%)$ & $8(19)$ & $25(51)$ & $<.01$ \\
\hline Prophylactic indomethacin, $\mathrm{n}(\%)$ & $14(33)$ & $39(80)$ & $<.01$ \\
\hline 5-min Apgar score & $8(6-8)$ & $7(5-7)$ & .15 \\
\hline Delivery room resuscitation & & & .02 \\
\hline Mask CPAP only, n (\%) & $3(7)$ & $12(24)$ & \\
\hline Endotracheal intubation, $\mathrm{n}(\%)$ & $40(93)$ & $37(76)$ & \\
\hline Intubation on the first day of life, $\mathrm{n}(\%)$ & $42(98)$ & $45(91)$ & .22 \\
\hline Score for Neonatal Acute Physiology II & $14(14-24)$ & $19(9-24.5)$ & .83 \\
\hline \multicolumn{4}{|l|}{ Postnatal characteristics before or at enrollment } \\
\hline Enrollment (day of life) & $26(19-31)$ & $27(21.5-31.5)$ & .39 \\
\hline Enrollment weight (g) & $987(856-1143)$ & $989(823-1173)$ & .82 \\
\hline PDA initial diagnosis (day of life) & $4(3-6)$ & $5(3-7)$ & .40 \\
\hline Any COXI treatment, n (\%) & $41(95)$ & $46(94)$ & 1.00 \\
\hline First COXI treatment (day of life) & $6(5-9)$ & $12(7-16)$ & $<.01$ \\
\hline Total COXI dose $(\mathrm{mg} / \mathrm{kg})^{*}$ & $1.2(1.2-1.5)$ & $1.4(0.75-1.5)$ & .84 \\
\hline PDA diameter at enrollment (mm) & $2.4(2.2-3)$ & $2.7(2.4-3.3)$ & .04 \\
\hline Celiac artery diastolic reversal at enrollment, $\mathrm{n}(\%)$ & $12(28)$ & $29(59)$ & $<.01$ \\
\hline Invasive ventilation at enrollment, $\mathrm{n}(\%)$ & $39(91)$ & $39(80)$ & .14 \\
\hline MAP at enrollment $\left(\mathrm{cmH}_{2} \mathrm{O}\right)$ & $10.7(3.0)$ & $11(3.0)$ & .66 \\
\hline Average daily MAP $\left(\mathrm{cmH}_{2} \mathrm{O}\right)$ & $10.9(8.8-12.2)$ & $10.0(8.8-11.3)$ & .16 \\
\hline Days of invasive ventilation at enrollment & $22.2(10.1)$ & $22.2(10.8)$ & 1.0 \\
\hline Percentage of invasive ventilation days at enrollment & $100(95-100)$ & $93(64-100)$ & .01 \\
\hline Pulmonary hemorrhage, n (\%) & $11(26)$ & $8(16)$ & .27 \\
\hline Inotropes for systemic hypotension, $\mathrm{n}(\%)$ & $11(26)$ & $18(37)$ & .32 \\
\hline Inhaled nitric oxide, $\mathrm{n}(\%)$ & $2(5)$ & $2(4)$ & .89 \\
\hline Grade 3 or 4 IVH, n (\%) & $6(14)$ & $16(33)$ & .04 \\
\hline Cystic periventricular leukomalacia, n (\%) & $3(7)$ & $2(4)$ & .54 \\
\hline Necrotizing enterocolitis stage $\geq 2, \mathrm{n}(\%)$ & $6(14)$ & $9(19)$ & .57 \\
\hline Sepsis, n $(\%)$ & $12(28)$ & $16(33)$ & .62 \\
\hline Systemic corticosteroids, n (\%) & $2(5)$ & $4(9)$ & .77 \\
\hline
\end{tabular}

Continuous data presented as mean (standard deviation) or median (IQR). GA, Gestational age; CPAP, continuous positive airway pressure; $P D A$, patent ductus arteriosus; COXI, cyclooxygenase inhibitor; MAP, mean airway pressure; IVH, intraventricular hemorrhage. *For infants treated with ibuprofen, the total dose of ibuprofen was converted to an equivalent total dose of indomethacin using the following calculation $(20 \mathrm{mg} / \mathrm{kg}$ ibuprofen, representing the dosing regimen used in our unit of a $10 \mathrm{mg} / \mathrm{kg}$ initial dose followed by 2 daily doses of $5 \mathrm{mg} / \mathrm{kg}=0.6 \mathrm{mg} / \mathrm{kg}$ indomethacin, representing 3 doses of $0.2 \mathrm{mg} / \mathrm{kg}$ administered every 12 hours).

fetal neuroprotection $[51 \%$ vs $19 \%, P<.01]$ and exclusively noninvasive delivery room respiratory support [24\% vs $7 \%, P=.02]$ ) (Table 1 ).

Infants in the acetaminophen group had a higher incidence of severe IVH (33\% vs $14 \%, P=.04)$, started COXI treatment later (day of life 12 vs $6, P<.01$ ), had a lower percentage of days of invasive mechanical ventilation at enrollment $(93 \%$ vs $100 \%, P=.01)$, and there were no differences in the day of life of PDA diagnosis, total dose of COXI treatment, or any other clinical characteristic
(Table 1). Infants in the acetaminophen group had increased echocardiography indices of ductal shunt volume at enrollment, including larger ductal diameter (2.7 vs $2.4 \mathrm{~mm}, P=.04$ ), and were more likely to have diastolic flow reversal in the celiac artery. Infants treated with acetaminophen (40 of 49 infants in the acetaminophen epoch) received a median of 7 days of therapy (IQR, 7-7) beginning at 26.5 days of life (IQR, 21-30.5; range, 1245). Surgically treated infants in the acetaminophen group underwent ligation at a later age compared with ligated 
TABLE 2. Neonatal outcomes according to epoch*

\begin{tabular}{|c|c|c|c|c|c|}
\hline Outcome & $\begin{array}{l}\text { Immediate surgical } \\
\text { ligation epoch } \\
(n=43)\end{array}$ & $\begin{array}{l}\text { Acetaminophen } \\
\text { epoch } \\
(n=49)\end{array}$ & $\begin{array}{c}\text { Crude OR } \\
(95 \% \text { CI })\end{array}$ & $\begin{array}{c}\text { Adjusted OR } \\
(95 \% \text { CI }) \dagger\end{array}$ & $\begin{array}{c}P \\
\text { value }\end{array}$ \\
\hline Patent ductus arteriosus ligation, $\mathrm{n}(\%)$ & $31(72)$ & $26(53)$ & $0.44(0.18-1.05)$ & $0.32(0.12-0.89)$ & .03 \\
\hline Death or CLD, n (\%) & $29(67)$ & $39(80)$ & $1.88(0.73-4.83)$ & $3.09(0.99-9.63)$ & .05 \\
\hline Death, n $(\%) \ddagger$ & $5(12)$ & $4(8)$ & $0.68(0.17-2.70)$ & $0.55(0.12-2.45)$ & .43 \\
\hline CLD, n (\%) & $25(58)$ & $36(73)$ & $1.83(0.72-4.69)$ & $3.34(1.05-10.58)$ & .04 \\
\hline Severe ROP, n (\%) & $4(9)$ & $6(12)$ & $1.31(0.34-5.03)$ & $1.73(0.38-7.86)$ & .48 \\
\hline Systemic corticosteroids, n (\%) & $6(14)$ & $10(20)$ & $1.58(0.52-4.80)$ & $2.74(0.71-10.58)$ & .14 \\
\hline
\end{tabular}

$O R$, Odds ratio; $C I$, confidence interval; $C L D$, chronic lung disease; $R O P$, retinopathy of prematurity. *Reference is the Immediate Surgical Ligation epoch. $\dagger$ Adjusted for $\mathrm{GA}$ and postnatal confounders representing illness severity/acquired morbidity at the time of enrollment (grade 3/4 intraventricular hemorrhage or cystic periventricular leukomalacia, percentage of days of mechanical ventilation, average daily MAP, and ductal diameter). †Defined as death before discharge home from the NICU.

infants in the earlier epoch (day of life 38 vs $28, P<.01$ ) and had longer duration of exposure to large PDA (41 vs 28 days, $P<.01)$.

\section{Main Outcomes}

Complete outcome information was available for all infants. Compared with infants in the immediate surgical ligation group, infants in the acetaminophen group had decreased surgical ligation $(26[53 \%]$ vs 31 [72\%], adjusted odds ratio [aOR] $0.32 ; 95 \%$ confidence interval [CI], 0.12-0.89; $P=.03)$, increased CLD (36 [73\%] vs 25 [58\%], aOR, 3.34; 95\% CI, 1.05-10.58; $P=.04$ ), and increased death or CLD of borderline significance (39 [80\%] vs 29 [67\%], aOR, 3.09; 95\% CI, 0.99-9.63; $P=.05)$. There was no difference between epochs in the odds of death before discharge or severe ROP requiring treatment (Table 2). Infants in the acetaminophen epoch took longer to successfully wean off of all positive pressure ventilation (28.5 vs 24 days after enrollment, aHR, 0.50; 95\% CI, 0.31-0.83; $P<.01$ ) (Table 3). Among the infants dependent on mechanical ventilation at enrollment $(\mathrm{n}=78)$, there was no difference in time to successful extubation (14 vs 11 days after enrollment, aHR, 0.73, 95\% CI, 0.44-1.19; $P=.21)$. Eleven infants $(15 \%)$ were reintubated at a median 26 days (IQR, 10-35) after successful extubation.

Among infants who underwent PDA surgery, there was no difference in the median time from referral for ligation to the date of surgery (epoch 1: 3 days [IQR, 2-6] vs epoch 2: 5 days [IQR, 2-8], $P=.12$ ). A minority of infants (12 in epoch $1 ; 11$ in epoch 2 ) were referred for but did not undergo surgery as a result of death (3 in epoch 1; 2 in epoch 2 ) or sufficient spontaneous ductal restriction or closure that the referral for ligation was canceled (9 in epoch 1; 9 in epoch 2).

A post hoc analysis was performed to determine if the increased CLD in the acetaminophen epoch was associated with a lack of response to acetaminophen treatment and prolongation of the duration of exposure to large ductal shunt volumes. However, there was no significant difference between responders and nonresponders to acetaminophen (defined as PDA closure or reduction to a small shunt on echocardiography) for the composite outcome of death or CLD (9/12 [75\%] in responders vs $21 / 27[78 \%$ ] in nonresponders, $P=.85$ ) or CLD alone (9/12 [75\%] in responders vs 19/27 [73\%] in nonresponders, $P=1.0$ ).

Among infants treated with ligation, the frequency of surgical complications was low in both epochs. There was no difference in operative mortality (3\% [epoch 1 ] vs $0 \%$ [epoch 2], $P=.46)$, postligation cardiac syndrome $(13 \%$ vs $8 \%, P=.50$ ), red blood cell transfusion within the first 24 postoperative hours ( $13 \%$ vs $4 \%, P=.12)$, chylothorax $(3 \%$ vs $0 \%, P=1.0)$, pneumothorax $(0 \%$ vs $8 \%, P=.2)$, or vocal cord paralysis $(0 \%$ vs $4 \%, P=.5)$.

\section{Complete Cohort}

The antenatal and postnatal characteristics of the broader cohort of infants 28 weeks or less gestation admitted to the NICU during the study period $(\mathrm{n}=467$ in epoch $1, \mathrm{n}=567$ in epoch 2) are presented in Table 4. Infants in epoch 2 had lower Score of Neonatal Acute Physiology II scores, were less likely to be exposed to chorioamnionitis, and were more likely to be exposed to antenatal corticosteroids. Pharmacologic treatment for PDA was less common among infants in epoch $2(24 \%$ vs $32 \%, P=.004)$, but there was no difference in the percentage of infants considered for surgical ligation $(8.6 \%$ in epoch 2 vs $9.2 \%$ in epoch 1 , $P=.75)$. Infants in epoch 2 had increased CLD (27\% vs $21 \%, P=.04)$. However, CLD was increased only among infants in epoch 2 without PDA, but not among infants who were diagnosed or treated for PDA (Table 4).

\section{DISCUSSION}

In this retrospective epoch study of preterm infants $28^{+6}$ or less weeks GA with persistent large PDA who were being considered for surgical ligation, late treatment with oral acetaminophen was associated with decreased odds of 
TABLE 3. Time to successful weaning from respiratory support

\begin{tabular}{|c|c|c|c|c|c|}
\hline Outcome & $\begin{array}{c}\text { Immediate surgical } \\
\text { ligation epoch }(n=43)\end{array}$ & $\begin{array}{l}\text { Acetaminophen } \\
\text { epoch }(n=49)\end{array}$ & $\begin{array}{c}\text { Crude HR } \\
(95 \% \text { CI })\end{array}$ & $\begin{array}{l}\text { Adjusted HR } \\
(\mathbf{9 5} \% \mathbf{C I})^{*}\end{array}$ & $P$ value \\
\hline $\begin{array}{l}\text { Time to wean off all positive pressure ventilation } \\
\text { (days after enrollment), median (IQR) } \dagger\end{array}$ & $24(15-30.5)$ & $28.5(15.5-36.5)$ & $0.74(0.48-1.15)$ & $0.50(0.31-0.83)$ & $<.01$ \\
\hline $\begin{array}{l}\text { Time to successful extubation } \\
\text { (days after enrollment), median (IQR) }\end{array}$ & $11(6-28)$ & $14(7-23)$ & $0.77(0.49-1.20)$ & $0.73(0.44-1.19)$ & .21 \\
\hline
\end{tabular}

Continuous data presented as mean (standard deviation) or median (IQR). $H R$, Hazard ratio; $C I$, confidence interval; IQR, interquartile range. *Adjusted for GA and postnatal confounders at the time of enrollment (grade 3/4 intraventricular hemorrhage or cystic periventricular leukomalacia, percentage of days of mechanical ventilation, average daily MAP, and ductal diameter). $\nmid$ Defined as survival without re-treatment with positive pressure ventilation for at least 7 days after discontinuation of positive pressure ventilation (invasive or noninvasive). $\ddagger$ Defined as survival without reintubation for at least 7 days after endotracheal extubation.

PDA surgery, but increased death or CLD of borderline significance, which was driven predominantly by an increase in CLD in the acetaminophen group. Infants in the acetaminophen group took longer to wean off of positive pressure ventilation than infants in the immediate surgical ligation epoch, but there was no difference in time to successful extubation.

No previous adjusted studies have evaluated the association of late acetaminophen therapy in preterm infants with clinical outcomes. Acetaminophen has been associated with ductal closure in uncontrolled studies of extremely preterm infants at risk of surgical ligation in case series comprising a total of 135 infants, in which ductal closure occurred in $74(55 \%)$ of treated infants. ${ }^{14,18-20}$ In addition, randomized trials of prophylactic or early treatment with acetaminophen reported high efficacy for ductal closure but no difference in mortality or respiratory outcomes when compared with COXI or placebo. ${ }^{7-10}$

In this study, the increased CLD and the longer duration of treatment with positive pressure ventilation in the acetaminophen group were unexpected findings given the evolution of clinical care over the study toward incorporating respiratory management strategies that reduce CLD. Infants in the acetaminophen group had a lower frequency of delivery room endotracheal intubation, reduced pre-enrollment invasive mechanical ventilation, decreased treatment with surgical ligation, and no difference in the frequency of treatment with systemic corticosteroids. Randomized trials have reported that the delivery room administration of primary nasal continuous positive airway pressure reduces the risk of death/CLD and CLD alone compared with endotracheal intubation. ${ }^{21}$ In addition, previous studies have associated a delayed, selective strategy for referring infants with persistent large PDA for surgical ligation with reduced CLD. ${ }^{22}$

The introduction of late acetaminophen treatment for PDA coincided with the adoption of a delayed and more selective pharmacologic treatment approach for PDA, which may have contributed to the increased CLD and longer duration of positive pressure ventilation in the second epoch. Among infants considered for surgical ligation, initial pharmacologic treatment occurred later in life in the acetaminophen group (12 vs 6 days of life, $P<.01$, Table 1), and these infants had a longer duration of exposure to large PDA (41 days in the acetaminophen epoch vs 28 days in the immediate surgical ligation epoch, $P<.01)$. A large ductal shunt increases pulmonary blood flow that may be injurious to the capillary endothelium and trigger an inflammatory cascade culminating in pulmonary edema, increased ventilator support, and the development of CLD. ${ }^{23,24}$ The optimal timing of PDA treatment, however, remains uncertain. Randomized controlled trials comparing early (first week of life) and late pharmacologic PDA treatment have consistently reported no difference in CLD or other respiratory outcomes. ${ }^{25,26}$ Yet prolonged exposure to large PDA has been associated with increased CLD, with each week of exposure resulting in an incrementally increased risk, possibly due to vascular remodeling from chronically increased pulmonary blood flow. ${ }^{27}$ This study adds to the body of evidence associating prolonged exposure to large PDA with adverse neonatal outcomes and suggests that treatment with oral acetaminophen to avoid surgical ligation in infants with prolonged persistent large PDA may not be the optimal strategy.

Although the secular trend toward more selective PDA treatment may have resulted in selection bias in assembling the cohort of infants in each epoch considered at risk of ligation, several findings in this study appear to mitigate the potential for such bias: First, although the decision to proceed with ligation was made at the discretion of the attending neonatologist (and potentially influenced by trends in management), it is unlikely that infants in epoch 1 had ductal shunts of marginal significance. The triaging and staging for ligation were performed in conjunction with the surgical ligation team at the quaternary surgical center, where the clinical and echocardiography diagnosis of persistent large PDA and rationale for ligation were reviewed before PDA surgery. All infants referred for ligation in both epochs had unrestrictive shunts, with $90 \%$ of infants having a ductal diameter greater than $2 \mathrm{~mm}$. Second, the percentage of infants considered for surgical ligation in each epoch was similar (Table 2), suggesting that the criteria for referral for surgical ligation 
TABLE 4. Antenatal and postnatal characteristics of the entire cohort of infants $\leq \mathbf{2 8}$ weeks in each epoch

\begin{tabular}{|c|c|c|c|}
\hline & Immediate surgical ligation epoch $(n=467)$ & Acetaminophen epoch $(n=567)$ & $P$ value \\
\hline \multicolumn{4}{|l|}{ Perinatal characteristics } \\
\hline GA at birth (wk) & $27(25,27)$ & $26(25,27)$ & .08 \\
\hline Birthweight (g) & $910(725,1089)$ & $880(720,1085)$ & .32 \\
\hline Female sex, n $(\%)$ & $210(45)$ & $263(46)$ & .43 \\
\hline Small for GA, n (\%) & $38(8)$ & $59(10)$ & .21 \\
\hline Multiple gestation, n (\%) & 127 (27) & $129(23)$ & .07 \\
\hline Pre-eclampsia, n (\%) & $97(21)$ & $87(15)$ & .05 \\
\hline Suspected chorioamnionitis, n (\%) & $81(17)$ & $66(12)$ & .009 \\
\hline Caesarean delivery, $\mathrm{n}(\%)$ & $272(58)$ & $315(56)$ & .21 \\
\hline Antenatal corticosteroids, $\mathrm{n}(\%)$ & $348(75)$ & $479(85)$ & $<.001$ \\
\hline 5-min Apgar score & $8(6,9)$ & $7(6,9)$ & .05 \\
\hline Surfactant treatment in first $48 \mathrm{~h}$ of life, $\mathrm{n}(\%)$ & $301(64)$ & $344(60)$ & .21 \\
\hline Score for Neonatal Acute Physiology II & $9(5,16)$ & $9(0,16)$ & .04 \\
\hline \multicolumn{4}{|l|}{ Neonatal characteristics } \\
\hline Diagnosis of PDA, n (\%) & $205(44)$ & $200(35)$ & .005 \\
\hline $\begin{array}{l}\text { Infants with diagnosis of PDA who did not receive } \\
\text { any COXI or surgical treatment, } \mathrm{n}(\%)\end{array}$ & $53 / 205(26)$ & $63 / 200(31)$ & .21 \\
\hline Any COXI treatment for PDA, n (\%) & $152(32)$ & $137(24)$ & .004 \\
\hline Acetaminophen treatment for PDA, $\mathrm{n}(\%)$ & $0(0)$ & $40(7)$ & $<.001$ \\
\hline Surgical ligation candidate, n (\%) & $43(9)$ & $49(9)$ & .75 \\
\hline Surgical ligation, n (\%) & $31(7)$ & $26(5)$ & .15 \\
\hline Any PDA treatment, $\mathrm{n}(\%)^{*}$ & $152(33)$ & $137(24)$ & .003 \\
\hline Grade 3 or 4 IVH, n (\%) & $42(9)$ & $64(11)$ & .23 \\
\hline Necrotizing enterocolitis stage $\geq 2, \mathrm{n}(\%)$ & $15(3)$ & $28(5)$ & .17 \\
\hline Death before discharge home, $\mathrm{n}(\%)$ & $43(9)$ & $47(8)$ & .60 \\
\hline \multicolumn{4}{|l|}{ Death or CLD, n (\%) } \\
\hline All infants & $133 / 467(28)$ & $188 / 567(33)$ & .11 \\
\hline Infants with diagnosis of PDA & $77 / 205(38)$ & $85 / 200(42)$ & .31 \\
\hline Infants with diagnosis of PDA, any treatment* & $63 / 152(41)$ & $68 / 137(50)$ & .16 \\
\hline Infants with diagnosis of PDA, no treatment & $14 / 53(26)$ & $17 / 63(27)$ & .94 \\
\hline Infants without a diagnosis of PDA & $56 / 262(21)$ & $103 / 367(28)$ & .06 \\
\hline $\begin{array}{l}\text { Infants who did not receive any PDA treatment } \\
\text { (with or without diagnosis of PDA) }\end{array}$ & $70 / 315(22)$ & $120 / 430(28)$ & .08 \\
\hline \multicolumn{4}{|l|}{ CLD (36 wk), n (\%) } \\
\hline All infants $\dagger$ & $90 / 422(21)$ & $141 / 521(27)$ & .04 \\
\hline Infants with diagnosis of PDA & $58 / 185(31)$ & $65 / 181(36)$ & .36 \\
\hline Infants with diagnosis of PDA, any treatment*, $\S$ & $51 / 139(37)$ & $53 / 123(43)$ & .29 \\
\hline Infants with diagnosis of PDA, no treatment $\|$ & $7 / 46(15)$ & $12 / 58(21)$ & .47 \\
\hline Infants without a diagnosis of PDA & $32 / 237(14)$ & $76 / 340(22)$ & .007 \\
\hline $\begin{array}{l}\text { Infants who did not receive any PDA treatment } \\
\text { (with or without diagnosis of PDA) }\end{array}$ & $39 / 283(14)$ & $88 / 398(22)$ & .006 \\
\hline
\end{tabular}

may have been consistent over the course of the study period.

\section{Study Strengths and Limitations}

The strengths of this study include the abstraction and adjustment for perinatal and postnatal pre-enrollment confounders and indices of illness severity, such as daily respiratory support, and echocardiography data, such as PDA size. This study is limited by being a small retrospective study with the potential for residual confounding due to unadjusted or unmeasured confounders, such as daily average $\mathrm{FIO}_{2}$ or other secular trends in NICU management that may have influenced clinical outcomes but that were not abstracted or identified. Second, an 
oxygen reduction test was not routinely performed, introducing the potential for bias in the ascertainment of CLD. In addition, we did not evaluate the association of epoch with neurodevelopmental outcomes, an important determination given the previously reported associations between ligation and neurodevelopmental impairment, and acetaminophen treatment with behavioral alterations in childhood such as autism spectrum disorder. ${ }^{28-31}$ Finally, the variation in outcomes across epochs due to acetaminophen treatment versus other concurrent secular trends in care is unknown. Prolonged exposure to large PDA, more selective criteria for referral for surgical ligation, and increased CLD among the broader cohort of infants in the second epoch may have influenced the differences in outcomes among the groups of infants in this study. A randomized trial of late acetaminophen treatment for persistent PDA among extremely preterm infants is required to provide an unbiased estimate of any benefit (or harm) of this therapeutic approach compared with immediate surgical ligation.

\section{CONCLUSIONS}

Late treatment with oral acetaminophen and avoidance of surgery for preterm infants with persistent large PDA were associated with reduced surgical ligation but increased CLD and prolonged treatment with positive-pressure ventilation. In light of a lack of improvement in clinical outcomes, the individual contributory effects of acetaminophen, surgical ligation, and prolonged exposure to PDA require further study to define the optimal approach.

\section{Conflict of Interest Statement}

Authors have nothing to disclose with regard to commercial support.

\section{References}

1. Sellmer A, Bjerre JV, Schmidt MR, McNamara PJ, Hjortdal VE, Host B, et al. Morbidity and mortality in preterm neonates with patent ductus arteriosus on day 3. Arch Dis Child Fetal Neonatal Ed. 2013;98:F505-10.

2. Kabra NS, Schmidt B, Roberts RS, Doyle LW, Papile L, Fanaroff A. Neurosensory impairment after surgical closure of patent ductus arteriosus in extremely low birth weight infants: results from the Trial of Indomethacin Prophylaxis in Preterms. J Pediatr. 2007;150:229-34.

3. Madan JC, Kendrick D, Hagadorn JI, Frantz ID III. Patent ductus arteriosus therapy: impact on neonatal and 18-month outcome. Pediatrics. 2009;123: 674-81.

4. Weisz DE, More K, McNamara PJ, Shah PS. PDA ligation and health outcomes: a meta-analysis. Pediatrics. 2014;133:e1024-46.

5. Lokku A, Mirea L, Lee SK, Shah PS. Canadian neonatal network. Trends and outcomes of patent ductus arteriosus treatment in very preterm infants in Canada. Am J Perinatol. 2017;34:441-50.

6. Hagadorn JI, Brownell EA, Trzaski JM, Johnson KR, Lainwala S, Campbell BT, Herbst KW. Trends and variation in management and outcomes of very lowbirth-weight infants with patent ductus arteriosus. Pediatr Res. 2016;80:785-92.

7. Dang D, Wang D, Zhang C, Zhou W, Zhou Q, Wu H. Comparison of oral paracetamol versus ibuprofen in premature infants with patent ductus arteriosus: a randomized controlled trial. PLoS One. 2013;8:e77888.

8. Oncel MY, Yurttutan S, Erdeve O, Uras N, Altug N, Oguz SS, et al. Oral paracetamol versus oral ibuprofen in the management of patent ductus arteriosus in preterm infants: a randomized controlled trial. J Pediatr. 2014;164:510-4.e1.
9. Dash SK, Kabra NS, Avasthi BS, Sharma SR, Padhi P, Ahmed J. Enteral paracetamol or intravenous indomethacin for closure of patent ductus arteriosus in preterm neonates: a randomized controlled trial. Indian Pediatr. 2015;52: 573-8.

10. Harkin P, Harma A, Aikio O, Valkama M, Leskinen M, Saarela T, et al. Paracetamol accelerates closure of the ductus arteriosus after premature birth: a randomized trial. J Pediatr. 2016;177:72-7.e2.

11. Ozdemir OM, Dogan M, Kucuktasci K, Ergin H, Sahin O. Paracetamol therapy for patent ductus arteriosus in premature infants: a chance before surgical ligation. Pediatr Cardiol. 2014;35:276-9.

12. Roofthooft DW, van Beynum IM, Helbing WA, Reiss IK, Simons SH. Paracetamol for ductus arteriosus closure: not always a success story. Neonatology. 2013;104:170.

13. El-Khuffash A, Jain A, Corcoran D, Shah PS, Hooper CW, Brown N, et al. Efficacy of paracetamol on patent ductus arteriosus closure may be dose dependent: evidence from human and murine studies. Pediatr Res. 2014;76:238-44.

14. Weisz DE, Martins FF, Nield LE, El-Khuffash A, Jain A, McNamara PJ. Acetaminophen to avoid surgical ligation in extremely low gestational age neonates with persistent hemodynamically significant patent ductus arteriosus. J Perinatol. 2016;36:649-53.

15. El-Khuffash AF, Jain A, McNamara PJ. Ligation of the patent ductus arteriosus in preterm infants: understanding the physiology. J Pediatr. 2013;162:1100-6.

16. Bell MJ, Ternberg JL, Feigin RD, Keating JP, Marshall R, Barton L, et al Neonatal necrotizing enterocolitis. Therapeutic decisions based upon clinical staging. Ann Surg. 1978;187:1-7.

17. Giaccone A, Jensen E, Davis P, Schmidt B. Definitions of extubation success in very premature infants: a systematic review. Arch Dis Child Fetal Neonatal Ed. 2014;99:F124-7.

18. Pharande P, Watson H, Tan K, Sehgal A. Oral paracetamol for patent ductus arteriosus rescue closure. Pediatr Cardiol. 2018;39:183-90.

19. Terrin G, Conte F, Oncel MY, Scipione A, McNamara PJ, Simons S, et al. Paracetamol for the treatment of patent ductus arteriosus in preterm neonates: a systematic review and meta-analysis. Arch Dis Child Fetal Neonatal Ed. 2016;101: F127-36.

20. Valerio E, Valente MR, Salvadori S, Frigo AC, Baraldi E, Lago P. Intravenous paracetamol for PDA closure in the preterm: a single-center experience. Eur $J$ Pediatr. 2016;175:953-66.

21. Schmolzer GM, Kumar M, Pichler G, Aziz K, O'Reilly M, Cheung PY. Non-invasive versus invasive respiratory support in preterm infants at birth: systematic review and meta-analysis. BMJ. 2013;347:f5980.

22. Jhaveri N, Moon-Grady A, Clyman RI. Early surgical ligation versus a conservative approach for management of patent ductus arteriosus that fails to close after indomethacin treatment. J Pediatr. 2010;157:381-7.

23. Alpan G, Scheerer R, Bland R, Clyman R. Patent ductus arteriosus increases lung fluid filtration in preterm lambs. Pediatr Res. 1991;30:616-21.

24. McCurnin D, Seidner S, Chang LY, Waleh N, Ikegami M, Petershack J, et al. Ibuprofen-induced patent ductus arteriosus closure: physiologic, histologic, and biochemical effects on the premature lung. Pediatrics. 2008;121:945-56.

25. Sosenko IRS, Fajardo MF, Claure N, Bancalari E. Timing of patent ductus arteriosus treatment and respiratory outcome in premature infants: a double-blind randomized controlled trial. J Pediatr. 2012;160:929-35.e1.

26. Van Overmeire B, Van De Broek H, Van Laer P, Weyler J, Vanhaesebrouck P. Early versus late indomethacin treatment for patent ductus arteriosus in premature infants with respiratory distress syndrome. J Pediatr. 2001;138:205-11.

27. Schena F, Francescato G, Cappelleri A, Picciolli I, Mayer A, Mosca F, et al. Association between hemodynamically significant patent ductus arteriosus and bronchopulmonary dysplasia. J Pediatr. 2015;166:1488-92.

28. Avella-Garcia CB, Julvez J, Fortuny J, Rebordosa C, Garcia-Esteban R, Galan IR, et al. Acetaminophen use in pregnancy and neurodevelopment: attention function and autism spectrum symptoms. Int J Epidemiol. 2016;45:1987-96.

29. Bauer AZ, Kriebel D. Prenatal and perinatal analgesic exposure and autism: an ecological link. Environ Health. 2013;12:41.

30. Andrade C. Use of acetaminophen (paracetamol) during pregnancy and the risk of autism spectrum disorder in the offspring. J Clin Psychiatry. 2016;77:e152-4.

31. Rebordosa C, Zelop CM, Kogevinas M, Sorensen HT, Olsen J. Use of acetaminophen during pregnancy and risk of preeclampsia, hypertensive and vascular disorders: a birth cohort study. J Mat Fet Neonatal Med. 2010;23:371-8.

Key Words: acetaminophen, bronchopulmonary dysplasia, ELGAN, mortality, PDA 